\title{
Aspects of Objectivity in Quantum Mechanics
}

\author{
Harvey R Brown \\ Sub-Faculty of Philosophy, University of Oxford, 10 Merton Street, Oxford OX1 4JJ, \\ U.K.
}

\section{Introduction.}

Relative to some inertial coordinate system defined in Galilean space-time, we assume that the quantum state of the physical system of interest satisfies the timedependent Schrödinger equation

$$
\mathrm{i} \frac{\mathrm{d}|\psi(t)\rangle}{\mathrm{d} t}=H|\psi(t)\rangle
$$

where the Hamiltonian $H$ may itself be time-dependent. (Here, and throughout this paper, units are chosen so that $\hbar=1$.) Suppose we require that (1) be covariant under some (possibly time-dependent) unitary transformation represented by $|\psi(t)\rangle \rightarrow\left|\psi^{\prime}\left(t^{\prime}\right)\right\rangle=U(t)|\psi(t)\rangle$ so that

$$
\mathrm{i} \frac{\mathrm{d}\left|\psi^{\prime}\left(t^{\prime}\right)\right\rangle}{\mathrm{d} t^{\prime}}=H^{\prime}\left|\psi^{\prime}\left(t^{\prime}\right)\right\rangle
$$

When $\mathrm{d} / \mathrm{d} t^{\prime}=\mathrm{d} / \mathrm{d} t$, it can easily be shown that covariance holds if and only if

$$
H^{\prime}=U H U^{-1}+\mathrm{i} \frac{\partial U}{\partial t} U^{-1}
$$

Recall that such covariance does not necessarily correspond to a symmetry of the Schrödinger dynamics: covariance of this general kind is expected to hold, for example, even for a wide class of non-linear coordinate transformations which are unitarily implementable. In such cases, the transformed Hamiltonian in (3) will not not 'take the same form' as does $H$ in (1). In the case of a transformation to a rectilinearly accelerating coordinate system, say, the scalar potential in $H^{\prime}$ will contain a new term corresponding to the inertial force acting on the particle-so that in particular a free particle no longer 'looks' free.

Something like the opposite of this familiar process can also occur. In very special cases of background potentials, such as that of a time-dependent simple 
harmonic potential, a quantum particle can exhibit free motion when described relative to an appropriately accelerating coordinate system ${ }^{1}$. In transforming in this case from an inertial coordinate system (with respect to which the potential is defined) to such a contrived non-inertial coordinate system, the energy spectrum of the particle goes from being purely discrete to purely continuous-a surprising state of affairs perhaps but consistent with non-invariance of the Hamiltonian as seen in (3) above. Even the existence of tunnelling in some cases turns out to be coordinatedependent. $^{2}$

Does this mean that it is not always an objective state of affairs as to whether a given particle is free, or whether tunnelling is taking place? This is similar to the question as to whether the Newtonian forces acting on a specific classical particle are not objective, given the ability to transform to the rest frame of the particle-relative to which obviously it 'moves freely'. The answer to both these questions is surely negative. To me, at any rate, the 'freedom' of the above-mentioned quantum particle when described relative to a contrived accelerating frame, for example, seems just as much of an artifact as the background thermal radiation 'seen'-via the Unruh effect—by a uniformly accelerating detector in the (inertially-defined) vacuum field in Minkowski spacetime. There is no doubt more to be said about this issue, but it will be skirted here. Questions of objectivity - in so far as they are concerned with the issue of coordinate-independence in Galilean spacetime-will be restricted in the paper to the context of differing inertial perspectives.

Section 2 contains a review of the covariance of the Schrödinger equation under local gauge transformations and Galilean coordinate transformations. In the subsection on gauge covariance, I include a brief discussion of the sense in which the gauge principle can be said to 'generate' dynamical gauge fields (such as the Maxwell field in the case of the $U(1)$ symmetry). The motivation of this discussion is two-fold. In part the discussion attempts to address the 'mystery' associated with the gauging procedure, recently highlighted by Teller. It also touches on the connection between gauge covariance in quantum theory and the requirement of general covariance in the general theory of relativity.

Before summarising the content of the subsequent sections, a few more introductory words are in order. Note that even in the case of the symmetries discussed below in Section 2, the second term on the RHS of (3) is generally of importance. Equation (3) forces us to be wary in accepting the common claim that under a unitary transformation $U$ implementing a symmetry, a self-adjoint operator $A$

\footnotetext{
${ }^{1}$ See Kuchar $\stackrel{\vee}{r}(1980)$, sections VII and VIII, and the independent work of Takagi (1990).

2 See Takagi (1991).
} 
representing some physical magnitude itself transforms unitarily as $A \rightarrow A^{\prime}=U A U^{-1}$. Such a transformation ensures of course that the mean value of $A^{\prime}$ (or rather its associated physical magnitude) defined with respect to the transformed state equals that of $A$ defined with respect to the original state. But in the case, say, of a Galilean coordinate transformation-where the associated unitary transformation of the state is time-dependent-equation (3) is telling us that $U H U^{-1}$ does not represent the same magnitude relative to the 'moving' observer as $H$ does relative to the 'stationary' one. Indeed, one wouldn't expect the total energy to be invariant under a passive Galilean boost, and it is far from being the only interesting magnitude for which the corresponding operator fails to transform unitarily in such cases as coordinate and gauge transformations. ${ }^{3}$

This state of affairs implies that a system undergoing Hamiltonian evolution which describes a closed loop in ray space (projective Hilbert space) relative to a given inertial coordinate system will generally fail to preserve this closure property when viewed from a (spatially) translated coordinate system if the translation is timedependent, and in particular when viewed from a boosted coordinate system. (A special case is the non-preservation of stationarity.) The ensuing issue of the Galilean coordinate-dependence of the geometric phase defined on paths in ray space will be taken up in Section 3 below. Some commentators have regarded such coordinate dependence as a defect in the standard formulation of geometric phase, but I am not so sure.

The existence of non-unitary, symmetry-related transformations of operators also raises a question as to the objectivity of 'sharp values' which a number of related interpretations of quantum mechanics attribute, qua elements of reality, to certain magnitudes of a system under certain conditions. In particular, the question as to whether sharpness imposed by the Eigenstate-Eigenvalue Link is invariant under coordinate transformations and/or gauge transformations is briefly examined in Section 4.

In the final section of the paper, attention is turned to the theory of quantum reference frames, due primarily to Aharonov and Kauffher. It would seem that this theory reformulates and reinforces the lesson urged in Section 4, namely that sharp values of observable magnitudes in quantum mechanics must, in a rather special (non-classical) sense, be given a relational, and not an absolute status. This, at least, is the conclusion of Section 5.

\footnotetext{
${ }^{3}$ For a discussion of the sense in which the 'common claim' above regarding the transformation $A \rightarrow A^{\prime}=U A U^{-1}$ is correct, see Brown and Holland (1998) and particularly Brown, Suarez and Bacciagaluppi (1998).
} 
The overlapping issues of symmetry and objectivity in quantum mechanics taken up in this paper-which is largely based on a number of recent collaborative efforts-reflect prominent themes in the work of Michael Redhead. Michael's influence on me over the last quarter-century as teacher, mentor, collaborator and friend has been enormous. It's with pleasure and gratitude that I dedicate the paper to him.

\section{Covariance of the Schrödinger equation.}

\section{(a) Gauge transformations.}

Consider the Schrödinger equation for a single, spinless particle evolving in the presence of a vector potential $\mathbf{A}(\mathbf{x}, t)$ and scalar potential $\mathrm{V}(\mathbf{x}, t)$, which may or may not be of electromagnetic origin ${ }^{4}$ :

$$
\mathrm{i} \frac{\partial \psi(\mathbf{x}, t)}{\partial t}=\left[\frac{-1}{2 m}\{\nabla-\mathrm{iA}(\mathbf{x}, t)\}^{2}+V(\mathbf{x}, t)\right] \psi(\mathbf{x}, t) .
$$

It is sometimes claimed that the form of the Hamiltonian in (4) is itself a direct consequence of Galilean invariance (or rather Galilean kinematics), a claim we shall briefly return to later ${ }^{5}$. In the meantime, let me repeat the well-known fact that (4) is covariant under a local gauge transformation $\psi(\mathbf{x}, t) \rightarrow \psi^{\prime}(\mathbf{x}, t)=\exp [\mathrm{i} \xi(\mathbf{x}, t)] \psi(\mathbf{x}, t)$ when the potentials transform as:

$$
\left.\begin{array}{l}
\mathbf{A}^{\prime}\left(\mathbf{x}^{\prime}, t^{\prime}\right)=\mathbf{A}(\mathbf{x}, t)+\nabla \xi(\mathbf{x}, t) \\
V^{\prime}\left(\mathbf{x}^{\prime}, t^{\prime}\right)=V(\mathbf{x}, t)-\frac{\partial \xi(\mathbf{x}, t)}{\partial t}
\end{array}\right\}
$$

In the case of Maxwell fields (where both (4) and the transformation of the wavefunction above hold in the case of a particle with unit charge), the transformations (5) leave electric and magnetic field strengths unaltered-even in the non-relativistic limit as we shall see in the next subsection.

4 An example of the appearance of a vector potential which is not related to an external magnetic field is mentioned in Section 5 below.

${ }^{5}$ See footnote 39 below. 
It is well-known too that the geometric role of the vector potential $\mathbf{A}$ is that of determining a connection, associated with the 'covariant' derivative $\mathbf{D} \equiv \nabla-\mathrm{iA}(\mathbf{x}){ }^{6}$ We can thus define the gauge-invariant quantity:

$$
f_{k l} \equiv \mathrm{i}\left[D_{k}, D_{l}\right]=\frac{\partial A_{l}}{\partial x_{k}}-\frac{\partial A_{k}}{\partial x_{l}}
$$

where $k, l=1,2,3$, which can be called the curvature tensor associated with the connection determined by $\mathbf{A}$. In the case where $\mathbf{A}$ has electromagnetic origin, $f_{k l}$ is non-zero wherever there exists a magnetic field-and it is detectable in quantum mechanics even when the particle is entirely excluded from the region where the magnetic field is confined, as was famously shown by Aharonov and Bohm in 1959. This result demonstrated the importance of interpreting the electromagnetic field as a gauge field corresponding to the local $U(1)$ group (see below).

Perhaps it is worth noting here that the relationship between the gauge principle and the introduction of a rule of parallel transport, and hence a connection, has also been usefully exploited for fibre bundles where the base space is other than physical space (or spacetime). In particular, it has been known for some time that the requirement of symmetrisation and antisymmetrisation of the states of collections of identical particles in quantum mechanics is intimately linked with the global topological structure of the reduced configuration space defined for $N$ particles

6 This geometric view of the potential is usually discussed in relation to the 4potential in relativistic quantum mechanics, to which we turn shortly. In case it needs stressing, the connection associated with this 4-potential, and the curvature it induces, are defined relative to the $U(1)$ fibre bundle whose base space is spacetime and whose fibres are the 'internal spaces' related to the local phases of the complex wavefunction. If one wants to say that such curvature is not a genuine curvature of spacetime, unlike that defined in general relativity, the reason can only be that the affine connection coefficients and the associated curvature tensor in the latter theory are defined with respect to the tangent bundle of spacetime. (Note that strictly speaking, in the case of the electromagnetic potential in quantum theory, both the covariant derivative operator and the connection coefficients actually depend on the charge of the particle as well as the 4-potential, so the connection depends on the type of particle involved. But the curvature operator-see equation (8) below-is defined to be charge-independent; see Lawrie (1990), § 8.1, for a nice introductory treatment of these issues.) 
moving in a three-dimensional physical space. ${ }^{7}$. (This reduced space is obtained by identifying points in the standard $3 \mathrm{~N}$-dimensional configuration space which are related by permutation of particle labels, and removing the singular points corresponding to spatial coincidence of two or more particles.) Suppose one introduces quadratically integrable wavefunctions subject to a local gauge symmetry on the reduced configuration space, which itself turns out to be doubly connected. Then it can be shown that the wavefunction must either change sign or remain invariant under an exchange of the particles. ${ }^{8}$ Furthermore, the former fermionic case is somewhat analogous to the force-free Aharonov-Bohm effect, but this time the 'gauge field' is confined to the off-limits singularities in the reduced configuration space. Now it may be open to interpretation just how explanatory this topological view of fermionic statistics is, but the overall approach seems to offer a deep insight into the range of possible statistics the particles may in principle exhibit ${ }^{9}$. The point I want to stress is that the local gauge principle on the reduced configuration space is central to the argument.

Returning to the familiar gauge fields on spacetime, I wish finally to comment on a claim sometimes made in the context of the relativistic quantum mechanics. This is the claim is that the gauge field (electromagnetism) has to be introduced to ensure covariance of the equation of motion under local $U(1)$ gauge transformations ${ }^{10}$. Now something almost magical seems to be occurring here, and one can sympathise with the puzzlement recently expressed by Teller (1997) at the apparent fact that in gauge theories generally, a change in mere "conventions" (local gauge) can have "dramatic repercussions in seeming to force the introduction of an otherwise neglected physical field!". The remaining comments in this subsection are offered in the hope of finding some alleviation of this puzzlement.

Let us consider then the case of a free spin- $1 / 2$ particle. As is well-known, the Dirac equation (in natural units wherein $c=\hbar=1$ )

\footnotetext{
${ }^{7}$ See in particular Leinaas and Myrheim (1977). For a brief review of this approach, and its particular naturalness in the context of de Broglie-Bohm pilot-wave theory, see Brown, Sjöqvist and Bacciagaluppi (1998).

8 The restriction to these two options does not hold if physical space is less than three-dimensional, allowing for the possibility of anyons in the two-dimensional case. 9 More recently, a derivation of the spin-statistics relation has resulted from the construction, within the same approach, of an exchange operator for identical particles with spin, with further topological significance in an enlarged Hilbert space; see Berry and Robbins (1997).

10 See, for example, Ryder (1987), p. 99.
} 


$$
\left(\mathrm{i} \gamma^{\mu} \partial_{\mu}-m\right) \psi(x)=0
$$

'becomes' gauge covariant under $\psi(x) \rightarrow \psi^{\prime}(x)=\exp [i \lambda \xi(x)] \psi(x)$ if the ordinary derivative $\partial_{\mu}$ acting on the spinorial wavefunction $\psi$ is replaced by the gaugecoviariant derivative $\mathrm{D}_{\mu}=\partial_{\mu}+i \lambda A_{\mu}(x)$-resulting in a relativistic analogue of (4). Here $\lambda$ is a 'coupling' constant (ultimately related to the charge of the particle, but at this point we can understand it merely as a factor such that $A_{\mu}$ need not have the same units as $\partial_{\mu}$ ), and the 4-vector field $A_{\mu}$ determines a connection on the $U(1)$ fibre bundle whose base manifold is spacetime. This vector field transforms as $A_{\mu}^{\prime}(x)=A_{\mu}(x)-\partial_{\mu} \xi(x)$. The very term 'minimal coupling' standardly associated with this procedure reflects the fact that the resulting equation of motion for the Dirac wavefunction is not the only conceivable gauge covariant equation which reduces to the original equation (7) when $A_{\mu}=0$, but it is the simplest.

But why should we be interested in a gauge-covariant version of (7) in the first place? Following Nakahara, we might relate the gauge principle to the truism that "physics should not depend on how we describe it", and compare it with the requirement of general covariance in the general theory of relativity ${ }^{11}$. Yet is it $a$ priori obvious that the choice of local phase of the wavefunction is merely a choice of description? Hardly. (I remain to be convinced even that the italicised claim above constitutes the decisive justification of general covariance in general relativity, but that's another story.) Surely it is hindsight, provided by the link with electromagnetism, that makes it appear $\mathrm{so}^{12}$. An entirely different issue, however, is the significance of introducing a connection, and hence a rule of parallel transport in the $U(1)$ bundle. It is this which tells us how to compare distant phases of the wavefunction, a natural requirement which does not, in itself, beg the question of gauge covariance.

What has actually been done above in replacing the ordinary derivative in (7) by the (gauge) covariant derivative is however analogous to the coordinate-general reformulation, in a flat affine spacetime, of the geodesic equation-essentially the equation of motion for a free classical particle-originally expressed in component form relative to a global inertial coordinate system (i.e., one in which the connection coefficients vanish everywhere). Again, this reformulation is carried out effectively by replacing the ordinary partial derivative by the covariant derivative associated with the affine connection defined on the tangent bundle. (For the sake of completeness, the details are given in the appendix.)

\footnotetext{
11 Nakahara (1990), p. 10.

${ }^{12}$ A related issue is why certain quantities in physics are gauged and not others (recently raised by Teller (1997), p. 517).
} 
In this case, the generally covariant equation of free motion captures the effect of 'inertial forces' acting on the particle from the perspective of a non-inertial coordinate system. In the case of the gauge covariant version of the free Dirac equation, one could perhaps say that the appearance of the 'potential' $A_{\mu}$ in the Dirac Hamiltonian similarly captures the effect of 'pure-gauge forces' arising out of the generalisation to arbitrary gauges. But in neither case is the connection a bona fide dynamical object yet, nor are the mentioned 'forces' of dynamical origin. This only comes about when (i) the connection gives rise to curvature, and (ii) when the matter field acts back on the connection.

Condition (i) is of course inconsistent with (7), in that there is no gauge in which $A_{\mu}=0$ everywhere (just as there are no global inertial coordinates systems when the curvature tensor in the spacetime tangent bundle is non-zero) ${ }^{13}$. The gaugeinvariant curvature is defined in terms of the commutator of the components of the covariant derivative (a generalisation of (6)):

$$
F_{\mu \nu} \equiv-\frac{\mathrm{i}}{\lambda}\left[\mathrm{D}_{\mu}, \mathrm{D}_{v}\right]=\partial_{\mu} A_{v}-\partial_{v} A_{\mu}
$$

Condition (ii) requires the introduction of an analogue of Einstein's field equations, which would determine inter alia the effect of the Dirac particle on the gauge potential $A_{\mu}$. Now if we view (7) as arising from an action principle, then a gauge-invariant action is already obtained just by replacing the ordinary derivative in the action associated with free motion by the gauge-covariant derivative. What is still lacking is a further gauge-invariant contribution to the action responsible for the back-action of the particle on the potential. The simplest Lorentz scalar that can be constructed from the gauge-invariant curvature is $F_{\mu \nu} F^{\mu \nu}$, and if, as is well-known, a term proportional to this scalar is added to the action, equations of motion for the gauge field are obtained which take the same form as Maxwell's equations when the electric current density $j^{\mu}(x)$ is interpreted as proportional to $\bar{\psi}(x) \gamma^{\mu} \psi(x)$, and the coupling constant $\lambda$ is related to electric charge. ${ }^{14}$

\footnotetext{
13 Once one introduces curvature along with minimal coupling, a weaker claim than Ryder's above can reasonably be made: "the electromagnetic properties of elementary fermions can be deduced simply by demanding that the Lagrangian be invariant under local phase transformations" (Collins et. al. (1989), p. 48). The argument here requires identifying the gauge potential with the known electromagnetic potential, and $\lambda$ with the charge of the fermion; the energy of the electromagnetic field is taken as given (op. cit. p. 49).

${ }^{14}$ See, for example, Lawrie (1990), p. 143.
} 
It is striking that in this important and suggestive 'derivation' of (classical) electrodynamics Nature seems to oblige in the sense of adhering both to the mimimal coupling procedure ${ }^{15}$ and the choice of the simplest gauge- and Lorentz-covariant contribution to the action that accounts for the dynamical properties of the gauge field. But the point I wish to stress is a different one: neither conditions (i) or (ii) above are consequences of the requirement of local gauge covariance. Condition (i) is motivated by physical phenomena like that of Aharonov and Bohm, which indicate that certain observable interference effects in quantum theory can be interpreted as anholonomies associated with the curvature of the gauge connection ${ }^{16}$. But the existence of such curvature-and hence of the electromagnetic field-is not strictly required by the process of constructing a gauge-covariant reformulation of (7) ${ }^{17}$. Condition (ii) is motivated by the action-reaction principle holding between matter and the gauge field ${ }^{18}$. Its analogue in general relativity is the requirement of the nonexistence of 'absolute objects' defined on the spacetime manifold (which are taken to act on the matter fields but are not acted upon), or what Wald ${ }^{19}$ has somewhat misleadingly called the 'principle of general covariance'. This principle is far stronger than the mere requirement that the field equations be written in general-covariant form (i.e. expressed in the tensor calculus) ${ }^{20}$, which is the tangent bundle analogue of gauge covariance.

These remarks should not be construed as disparaging the heuristic importance of treating electromagnetism as a gauge theory, which is of course

\footnotetext{
${ }^{15}$ For a discussion of the electromagnetic significance of minimal coupling, and some subtleties in its definition that have been overlooked here, see Sakurai (1964), pp. 182-3.
}

16 Although the Aharonov-Bohm (AB) effect was instrumental in the recognition of electromagnetism as a gauge field in quantum theory, the consideration here does not depend on the gauge curvature being inaccessible to the quantum system as in the $A B$ effect.

17 Note that Rai Dastidar and Rai Dastidar (1994, 1995) have already argued that local gauge invariance in quantum theory does not imply the existence of an external electromagnetic field, but they do not (I think) make it clear that the gauge potentials introduced into their gauge invariant formulation of the quantum dynamics in the case of free particles correspond to a flat connection.

18 See Lawrie (1990), p. 161, and Anandan (1997), section 5.4.. For a wider discussion of the role of the action-reaction principle in modern physics, see Anandan and Brown (1995).

19 Wald (1984), p. 57.

${ }^{20}$ For a fuller discussion of this point, see Brown and Sypel (1995), section 4. 
illustrated in the successful use of the gauge principle in the standard model of particle physics. The postulation of physical gauge fields such as the gluon field in chromodynamics was made possible by the earlier recognition of the gauge structure of the electromagnetic field, and the pioneering investigation in 1954 of possible gauge fields associated with the $S U(2)$ group by Yang and Mills. But as before, direct observable effects of the gauge potentials in question can in principle be expected only under the assumption that the gauge fields-the generalisations of (8) for nonAbelian gauge groups-are non-vanishing and satisfy the action-reaction principle.

We shall return to the issue of gauge invariance and some of its implications in quantum mechanics in Section 4.

(b) Galilean covariance. ${ }^{21}$ Let us consider a passive coordinate transformation from the frame $F$ (relative to which (4) holds) to the frame $F^{\prime}$ moving at uniform velocity $\mathbf{v}$ relative to $F$ :

$$
\mathbf{x}^{\prime}=\mathbf{x}-\mathbf{v} t, \quad t^{\prime}=t
$$

Relative to $F^{\prime}$, the value of the wavefunction at an arbitrary space-time location is related to that of $\psi$ at the same location by a phase factor

$$
\psi^{\prime}\left(\mathbf{x}^{\prime}, t^{\prime}\right)=e^{i \phi} \psi(\mathbf{x}, t)
$$

in order to ensure invariance of the probability density at that location. Textbook treatments $^{22}$ of the Galilean covariance of the Schrödinger equation invariably deal with the special cases of a free particle or one with finite scalar potential $V$, where it is shown that covariance is secured, assuming $V$ transforms as a scalar field, when

$$
\phi=m\left(\mathbf{v}^{2} t / 2-\mathbf{v} \cdot \mathbf{x}\right) .
$$

However, since we do not expect the phase in (10) to depend on the dynamics, we expect it to be independent of the vector potential $\mathbf{A}$, as well as $V$. Indeed, it can

\footnotetext{
21 This subsection contains a brief summary of the detailed review of the Galilean covariance of quantum mechanics given in Brown and Holland (1998).
}

${ }^{22}$ See, for example, Ballentine (1990, section 4.3) 
easily be shown ${ }^{23}$ that (4) is Galilean-covariant under (9), (10) and (11) when the potentials transform as

$$
\left.\begin{array}{l}
\mathbf{A}^{\prime}\left(\mathbf{x}^{\prime}, t^{\prime}\right)=\mathbf{A}(\mathbf{x}, t) \\
V^{\prime}\left(\mathbf{x}^{\prime}, t^{\prime}\right)=V(\mathbf{x}, t)-\mathbf{v} \cdot \mathbf{A}(\mathbf{x}, t)
\end{array}\right\}
$$

(In fact (11) and (12) are necessary conditions for the covariance of (4), ignoring irrelevant gauge transformations and arbitrary constants in the phase (11).) We see that in the general case, the scalar potential $V$ no longer transforms as a scalar field.

To see how (11) involves a gauge transformation ${ }^{24}$ —although as is stressed at the end of this subsection, a Galilean transformation is more than just a gauge transformation-let us briefly consider what happens when we suppose that the wavefunction transforms like a scalar field, which we write as $\psi(\mathbf{x}, t) \rightarrow \varphi^{\prime}\left(\mathbf{x}^{\prime}, t^{\prime}\right)=\psi(\mathbf{x}, t)$. In this case, still using (12), one obtains

$$
\mathrm{i} \frac{\partial \varphi^{\prime}}{\partial t^{\prime}}=\left[\frac{-1}{2 m}\left\{\nabla^{\prime}-\mathrm{i}\left(\mathbf{A}^{\prime}+m \mathbf{v}\right)\right\}^{2}+V^{\prime}-m \mathbf{v}^{2} / 2\right] \varphi^{\prime}
$$

Here we see that in relation to (4), the transformed Hamiltonian picks up a curlfree-and hence uninteresting-vector potential $m \mathbf{v}$ (itself a case of a flat connection), as well as the extra constant term $-m \mathbf{v}^{2} / 2$. It is largely a matter of convenience whether we absorb this latter term into $V^{\prime}$, but at any rate both terms can now be eliminated by the appropriate gauge transformation of $\varphi^{\prime}$ :

$$
\left.\begin{array}{rl}
\psi^{\prime}\left(\mathbf{x}^{\prime}, t^{\prime}\right) & \equiv \exp \left[\operatorname{iim}\left(-\mathbf{v} \cdot \mathbf{x}^{\prime}-\mathbf{v}^{2} t^{\prime} / 2\right)\right] \varphi^{\prime}\left(\mathbf{x}^{\prime}, t^{\prime}\right) \\
& =\exp \left[\operatorname{im}\left(-\mathbf{v} \cdot \mathbf{x}+\mathbf{v}^{2} t / 2\right)\right] \psi(\mathbf{x}, t)
\end{array}\right\}
$$

thus recovering the phase in (11) and ensuring the covariant form of the waveequation for $\psi^{\prime}\left(\mathbf{x}^{\prime}, t^{\prime}\right) .25$

23 See Takagi (1991, p. 465) and Brown and Holland (1998). It is perhaps surprising that the demonstration of covariance in the general case (involving the vector as well as scalar potential) is so rare in the literature.

24 The following argument is taken from Takagi (1991, p. 465), where the treatment involves the more general case of a time-dependent $\mathbf{v}$.

25 Note that (11) ensures the covariance of the fundamental de Broglie relation between momentum and wavelength in the case of plane wave solutions of (4) when 
It is worth noting that one can find a set of electromagnetic 'field' equations, which can be considered the non-relativistic limit of Maxwell's equations in the case where magnetic effects predominate over electric ones, which are strictly Galilean covariant under (12). ${ }^{26}$ (The field equations are identical to Maxwell's except for the absence of the displacement current: time-varying electric fields do not induce a magnetic field. The result is a phenomenological theory of magnetostatics, corresponding to the usual macroscopic situation where negative and positive charges cancel.) The electric and magnetic fields are related to the scalar and vector potentials in the usual way. Hence a fully Galilean-covariant quantum theory of the Schrödinger field interacting with an external electromagnetic field is possible. It should however be emphasised here that a necessary condition for the covariance of this theory is that the Schrödinger current and charge density do not act as sources of the Maxwell field.

The results reviewed so far allow us to analyse the problem of covariance in the de Broglie-Bohm (de B-B) pilot-wave interpretation of quantum mechanics. Recall that the guidance equation for the de B-B corpuscle takes the following form when the system is in the presence of a vector potential $\mathbf{A}^{27}$ :

$$
m \dot{\mathbf{x}}=\nabla S-\mathbf{A}
$$

where $S$ is the phase of the 'guiding field': $\psi(\mathbf{x}, t)=R(\mathbf{x}, t) \exp [\mathrm{i} S(\mathbf{x}, t)]$. (Again, if $\mathbf{A}$ is of electromagnetic origin, (15) holds when the particle has unit charge.) Here, as in (4), the role of the vector potential can be seen as a compensating term: it renders the velocity of the corpuscle invariant under local gauge transformations, as the reader can easily check.

But is (15) consistent with Galilean kinematics? From (11) and (12) we see that

the potentials are identically zero. The fact that wavelength, like momentum, does not transform invariantly under a Galilean transformation indicates that the wavefunction is quite different from a classical wave amplitude, a fact which is frequently forgotten when appeal is made in quantum mechanics to the Bohrian complementarity between the 'classical' pictures of wave and particle. For further details, see Lévy-Leblond (1976).

26 These field equations correspond to the 'magnetic' (nonrelativistic) limit of Maxwell's equations formulated in Le Bellac and Lévy-Leblond (1973). It is shown in this work that there are two natural nonrelativistic limits, which makes it difficult to say which electrodynamic effects are strictly relativistic in nature.

27 See Holland (1993). 


$$
\begin{aligned}
m \dot{\mathbf{x}}^{\prime} & =\nabla^{\prime} S^{\prime}-\mathbf{A}^{\prime} \\
& =\nabla S-m \mathbf{v}-\mathbf{A} \\
& =m \dot{\mathbf{x}}-m \mathbf{v},
\end{aligned}
$$

so we recover the Galilean transformation of a momentum vector. Furthermore, it can be shown from (12) that the classical Lorentz force acting on the de B-B corpuscle when immersed in an electromagnetic field (which exists as well as the force due to the Bohm quantum potential-itself also being affected by the external field) transforms invariantly, as one expects of a classical force in Galilean space-time.

This state of affairs might appear comforting. But for those who regard the true de B-B dynamics as captured in the first-order equation (15), the forces acting on the corpuscle and generated by the guiding wave are Aristotelian, not Newtonian: they produce velocities not accelerations. This entails that there is a natural state of 'motion', which is rest. Yet no privileged frame is picked out by the 'hidden' dynamics of the corpuscle, and what may at first sight have looked like a sign of strength of the theory is now seen as a possible source of embarrassment. ${ }^{28}$

We finish this brief review of Galilean covariance with a glance at the state of affairs in the abstract formalism, since we will refer to some of the details later. Here we replace (4) by

$$
\left.\mathrm{i} \frac{d|\psi(t)\rangle}{d t}=\left[\frac{\{\mathbf{P}-\mathbf{A}(\mathbf{Q})\}^{2}}{2 m}+V(\mathbf{Q}, t)\right] \psi(t)\right\rangle
$$

where $\mathbf{P}$ is the operator representing the canonical momentum and $\mathbf{Q}$ is again the position operator. (We omit putting hats on symbols representing operators.) Equation (17) is covariant under a passive Galilean boost implemented by a unitary transformation, represented by $|\psi(t)\rangle \rightarrow\left|\psi^{\prime}\left(t^{\prime}\right)\right\rangle=U_{G}|\psi(t)\rangle$, when $\mathbf{P}$ and $\mathbf{Q}$ transform invariantly-note, not unitarily — and

$$
\left.\begin{array}{l}
\mathbf{A}^{\prime}\left(\mathbf{Q}^{\prime}, t^{\prime}\right)=U_{G}[\mathbf{A}(\mathbf{Q}, t)] U_{G}{ }^{-1} \\
V^{\prime}\left(\mathbf{Q}^{\prime}, t^{\prime}\right)=U_{G}[V(\mathbf{Q}, t)-\mathbf{v} \cdot \mathbf{A}(\mathbf{Q}, t)] U_{G}^{-1}
\end{array}\right\}
$$

and when

\footnotetext{
${ }^{28}$ For further discussion of this issue, see Brown et al. (1996) and Valentini (1997).
} 


$$
U_{G}=\exp \left(\mathrm{i} m \mathbf{v}^{2} t / 2\right) \exp (\mathrm{iv} \cdot \mathbf{P} t) \exp (-\mathrm{i} m \mathbf{v} \cdot \mathbf{Q}) .
$$

The form of (19) $)^{29}$ should come as no surprise, given (13). The 'extra' factor in (18) containing $\mathbf{P}$ simply accounts for the fact that a passive Galilean transformation involves a time-dependent translation of the coordinate axes (as well as a velocity transformation). This is already taken into account in (9) where we are comparing the values of the primed and unprimed wavefunctions at the same space-time location, or in other words for different arguments (coordinates)-which also acounts for the absence of the unitary factors in (11) when compared with (17). (It is this feature that makes a passive boost something beyond a mere gauge transformation.) The unitary operator implementing an active boost of the particle plus potentials is given by the inverse of (17), and is of course consistent with the role of the canonical operators -P and $\mathbf{Q}$ as generators in quantum mechanics of active translations and (instantaneous) boosts respectively.

\section{The non-invariance of geometric phase.}

Following the seminal work of Berry (1984) on systems undergoing cyclic adiabatic evolution, it has come to be realised that there exists an important geometrical structure in the quantum formalism related to the phase of a quantum system undergoing Schrödinger evolution. Let us consider a system undergoing cyclic (not necessarily adiabatic) evolution, so that during the temporal interval $[0, T]$, the system's final and initial states coincide up to a phase factor: $|\psi(T)\rangle=\exp (\mathrm{i} \phi)|\psi(0)\rangle$, where $\phi$ is an arbitrary real number. When projected onto ray space, i.e. the projective Hilbert space $\wp$, this evolution defines a closed path. Now suppose we have the idea of subtracting from the total phase $\phi$ the accumulation of local phase changes produced by the motion on this path. By 'a local phase change' is meant the quantity $\delta \phi\left(\psi_{t}, \psi_{t+\delta t}\right)=-\mathrm{i}\langle\psi(t)|\mathrm{d} / \mathrm{d} t| \psi(t)\rangle \delta t$. We are subtracting then from the total phase the quantity

\footnotetext{
${ }^{29}$ See also Fonda and Ghirardi $(1970, \S 2.5)$. Note that (19) is independent of the dynamics: the potentials in the Hamiltonian in (17) make no appearance. This is to be expected given the properties of Galilean space-time. However, the same situation will not hold for the analogue of (19) in relativistic quantum mechanics given the relativity of simultaneity in Minkowski space-time.
} 


$$
\left.\begin{array}{rl}
\phi_{d} & \left.\equiv-\mathrm{i} \int_{0}^{T}\langle\psi(t)|\mathrm{d} / \mathrm{d} t| \psi(t)\rangle \mathrm{d} t\right\rangle \\
& =-\int_{0}^{T}\{\psi(t)|H| \psi(t)\rangle \mathrm{d} t
\end{array}\right\}
$$

where $H$ is again the Hamiltonian responsible for the cyclic motion (still putting $\hbar=1)$. Because it depends on $H$, the quantity $\phi_{d}$ is called the dynamical phase. Now what we are left with after the subtraction, $\phi_{g}=\phi-\phi_{d}$, is the 'geometric phase', formulated by Aharonov and Anandan (1987). It is reparametrisation invariant (i.e. independent of the speed at which the path in $\wp$ is traversed). Moreover, it takes the same value for all the (infinity of) evolutions in the Hilbert space which project onto the given closed path in $\wp$; it is a property only of that path. It is natural then to interpret it as the anholonomy associated with 'parallel transport'-transport in which there is no local phase change-around the closed curve in $\wp$. The existence of geometric phase testifies to the existence of a non-flat connection on $\wp$.

I shall return at the end of this section to the significance of the discovery of the curved geometry of the ray space in quantum mechanics. At this point I wish to mention a recent result concerning the Galilean non-invariance of geometric phase.

A curve in space-time is a geometrical object; in particular whether it is closed or not does not depend on the choice of coordinate system. Now imagine a perfectly elastic ball bouncing on the hard floor of a laboratory; to an observer at rest relative to the lab, the evolution of the ball defines a closed path in state (phase) space. Yet to an observer in uniform motion relative to the lab, it doesn't: the ball does not return to the same spatial location at each bounce. ${ }^{30}$ Note that it is not the speed per se of the moving observer that counts here, but rather the fact that the new frame is spatially translated in a time-dependent way relative to the lab frame. Analogously, we should not expect in quantum mechanics that the closure property of curves in $\wp$ should be invariant under time-dependent unitary transformations of the state. In particular, we should expect, and indeed it is so, that closure is (inertial-) frame-dependent, given the time-dependence of the second exponent in (19) — which, as noted above, has to do with passive time-dependent translations and not (instantaneous) boosts per se.

The fact that the very condition for the definability of geometric phase (closure of the path in $\wp$ ) is not generally preserved under time-dependent unitary transformations (including gauge transformations) was recognised from the start. Indeed, it was recognised that even in the special cases (which exclude unitary

\footnotetext{
${ }^{30}$ I thank Jeeva Anandan for this argument.
} 
implementations of Galilean transformations) where closure is preserved, the geometric phase is still not invariant ${ }^{31}$. However, an interesting development - particularly from the point of view of Galilean transformations-had to do with the formulation of a geometric phase factor $\gamma_{g}$ for open paths in $\wp$ given independently by Aitchison and Wanelik (1992) and Mukunda and Simon (1993), and which is defined by

$$
\exp \left(\mathrm{i} \gamma_{g}\right)=\left(\frac{\{\psi(0)|\psi(T)\rangle}{\langle\psi(T) \mid \psi(0)\rangle}\right)^{1 / 2} \exp \left(-\int_{0}^{T}\langle\psi(t)|H| \psi(t)\rangle \mathrm{d} t\right)
$$

for evolutions such that $\left\{\psi(T)|\psi(0)\rangle \neq 0\right.$. The new phase $\gamma_{g}$ is also projectivegeometric and reduces to the Aharonov-Anandan phase $\phi_{g}$ in the case of cyclicity. In the case of an arbitrary open curve in $\wp, \gamma_{g}$, when it is well-defined, is equal to $\phi_{g}$ defined on the geodesic closure of this curve-geodesics being defined of course relative to the mentioned connection on $\wp$.

We may now ask whether $\gamma_{g}$ is Galilean-invariant. In particular, suppose there is some gauge such that relative to the frame $F$ the curve $C$ in $\wp$ defined by the Schrödinger evolution of the system in the interval $[0, T]$ is closed. A passive Galilean transformation to the frame $F^{\prime}$ implemented by (19) will transform $C$ into an open curve $C^{\prime}$. Then is the geometric phase $\phi_{g}=\gamma_{g}$ for $C$ equal to $\gamma_{g}$ for $C^{\prime}$ (or equivalently $\phi_{g}$ for the geodesic closure of $C^{\prime}$ )? The answer is no, and again the guilty party is the time-dependent exponent containing the canonical momentum $\mathbf{P}$ in $(19)^{32}$.

Intuitively, the non-invariance result is perhaps not surprising. A timedependent translation of the coordinate axes (and hence a pure Galilean transformation) will, as we have seen, transform a closed curve in $\wp$ into an open one, whose geodesic closure in turn is a different closed curve to the original one. After all, curves in $\wp$ are geometric objects, but not in space-time. Given a rule for parallel transport in $\wp$, why should we expect the anholonomy-the geometric phase - to be the same on both of these distinct closed curves? It is the curvature of $\wp$ that is the relevant invariant entity, and this being the case we expect different anholonomies produced by parallel transport on different closed curves in the space.

Note that some commentators appear to regard the non-invariance result as representing a weakness in current formulations of geometric phase; indeed some

\footnotetext{
${ }^{31}$ See Anandan (1989).

32 See Sjöqvist et al. (1997).
} 
attempts to remedy the situation have been undertaken ${ }^{33}$. However, the considerations expressed in the last paragraph, together with the fact that geometric phase has been experimentally 'observed'-even in cases not involving spin states which are Galilean invariant ${ }^{34}$ - tend in my opinion to cast doubt on the necessity of an invariant reformulation of geometric phase. No doubt the issue deserves further analysis, but I leave it here.

I return now to the question of the ultimate significance of the discovery of the curved geometry of $\wp$. (I accept of course that Berry's original 1984 discovery was genuinely surprising; it was generally assumed at the time that the total phase acquired in cyclic adiabatic evolution is purely dynamical. ${ }^{35}$ The question I am now raising is: given the existence of geometric phases in this case and in the general case of Aharonov and Anandan, what is its significance?) The recognition that there is a feature of Schrödinger evolution that is indifferent to the dynamical details specified by the Hamiltonian — or at least the choice of Hamiltonian within an infinite relevant class-, and that depends on only the fixed path in $\wp$ has led to a significant geometrical reformulation of quantum mechanics. In particular, the symplectic structure of $\wp$, and its role as a metric space have been clarified, leading inter alia to a new insight into the energy-time uncertainty relations ${ }^{36}$. The situation here is reminiscent of the discovery by Minkowski of the geometric formulation of special relativity, and the structure of Minkowski space-time ${ }^{37}$. But in both cases, no new predictions are involved. (The interference effects that are involved in actual 'measurements' of geometric phase are of course predictable on the basis of good, old Schrödinger dynamics.) Indeed, the real significance of Minkowski's contributions to relativity theory only came to be seen in the later success of Einstein's general theory, where the space-time geometry itself became a dynamical player. Analogously, the

33 A Galilean- (but not gauge-) invariant formulation of geometric phase was given in García de Polavieja (1997), and subsequently Bacciagaluppi (1997) has produced a formulation which is both Galilean- and gauge-invariant, defined on a bundle incorporating time and the ray space. An unsettling feature of the latter formulation is that the connection now depends on the scalar potential $V$ in the Hamiltonian.

${ }^{34}$ For more details, see Sjöqvist et al. (1997).

35 Even after Berry's discovery, it continued to be widely supposed that the geometrical phase associated with non-cyclic abiabatic motion could be ignored ('gauged away'), but this has been shown to be erroneous; see García de Polavieja and Sjöqvist (1998).

${ }^{36}$ See Anandan (1991).

37 I have compared the 'causal' properties, or rather the lack thereof, of both Minkowski space-time and the projective Hilbert space in Brown (1996). 
full significance of geometric phase may prove to be in its heuristic power in a future reformulation of quantum principles ${ }^{38}$.

\section{Objectivity of sharp values?}

A variety of interpretations of quantum mechanics seek to augment the standard state description of a quantum system (be the state pure or mixed) by specifying state-dependent rules for assigning sharp values to some of the self-adjoint operators representing magnitudes (or equivalently for assigning bivalent truth values to some propositions describing properties of the system). In what sense do these values (or truth-values) represent or reflect the existence of objective elements of reality? In this section, I shall briefly summarise some of the considerations contained in a recent attempt to answer this question ${ }^{39}$.

Let us consider those interpretations whose value-assignment rules coincide with the Eigenstate-Eigenvalue Link (EEL) for systems which for some suitable period of time may be regarded as unentangled with the environment. (This includes some prominent versions of the so-called 'modal' interpretation.) To fix our ideas, let us imagine the free particle $p$ in the original 1935 Einstein-Podolsky-Rosen argument, whose separated twin particle-originally entangled with $p$-has just undergone a measurement of the $x$-component of momentum $P_{x}$. (Recall that the initial EPR state of the two particles is an eigenstate of total momentum in a direction we associate with the $x$-axis.) Using either the EPR criterion of reality, or the stronger EEL (since we may effectively consider $p$ to be in a pure (improper) eigenstate of momentum as a result of the distant measurement), $P_{x}$ is assigned a sharp value for $p$. Classically, momentum is of course frame-dependent; classical momentum is a relational property involving the body in question and a given inertial coordinate system (or a family thereof adapted to a given inertial frame) and under a passive Galilean transformation it changes its sharp value as in (16) above. In quantum mechanics, the canonical operators $\mathbf{P}$ and $\mathbf{Q}$ can be taken to transform invariantly under a Galilean transformation (as was mentioned in Section $2 \mathrm{~b}$ above) and it transpires, unsurprisingly, that if $P_{x}$ is sharp for $p$ relative to the EPR lab frame, it is also sharp for $p$ relative to a moving frame-the value having undergone the usual Galilean transformation for momentum. An exactly analogous situation holds for the position operator $\mathbf{Q}$, as well as for the velocity operator $\dot{Q}_{i} \equiv \mathrm{i}\left[H, Q_{i}\right], i=x, y, z$, where $H$ is again the Hamiltonian; indeed it is so even when the system is not free (and hence

\footnotetext{
${ }^{38}$ See the concluding remarks in Anandan $(1991 ; 1992)$.

39 See Brown, Suárez and Bacciagaluppi (1998).
} 
when the velocity operator is not proportional to the corresponding component of canonical momentum, failing thereby to transform invariantly ${ }^{40}$ ).

In all these cases, 'sharp values', when they obtain under EEL, are coordinatedependent, and hence if construed as objective elements of reality, should presumably be regarded not as intrinsic properties of the quantum system but relational properties, analogously to their classical counterparts. (So far we are treating the properties as defined relative to inertial coordinate systems, but as is emphasised in the next section, this is arguably somewhat unrealistic from an operational point of view. For the moment, however, let's stick to this viewpoint.) Note however that when the particle is being acted upon by an external field that contributes a vector potential to the Hamiltonian, the canonical momentum is gauge-dependent. Classically, its value changes under a gauge transformation; but in quantum mechanics, the very sharpness of a given component of momentum is generally not preserved under a gauge transformation. Sharpness itself is not gauge-independent in quantum mechanics, a state of affairs that can also occur for the total energy when the gauge transformation is time-dependent.

This non-invariance of sharpness is exhibited in quantum mechanics also in the case of Galilean transformations, one example being that of orbital angular momentum (whose corresponding operator has a discrete spectrum and is therefore a more satisfactory object for the application of EEL than the magnitudes being discussed so far.) A sharp component of angular momentum will lose its sharpness even when the spatial direction along which it is defined is orthogonal to the direction of the boost, and even at the instant when the systems of spatial axes associated with

40 Given the Hamiltonian in (4) and (16), the velocity operator transforms invariantly (i.e. $\dot{Q}_{i}{ }^{\prime}=\dot{Q}_{i}$ ) normally only at the initial instant when the two coordinate systems associated with a Galilean transformation coincide. It is worth noting here that a derivation of this generic Hamiltonian based on Galilean kinematics was provided by Jauch (1964), and has since repeated on a number of occasions in the literature. Jauch's theorem rests on inter alia the very assumption that $\dot{Q}_{i}{ }^{\prime}=\dot{Q}_{i}$ at $t=t^{\prime}=0$. Doubts about the a priori validity of this assumption, as well as about further details of the Jauch theorem, are found in Brown and Holland (1998). On a more general note, it can be argued that Jauch's approach, which uses the fundamental properties of space and time to constrain the form of the Hamiltonian, is pointing in the wrong direction. Space-time structure itself may be seen as a consequence of the symmetries of the dynamics of quantum systems, and not as fundamental. This view is consistent with the profound analysis of the role of geometry in physical theories given in Anandan (1980); its implications in the case of quantum theory are further explored in Anandan (1997, particularly chapter 5). 
the two frames coincide. Note incidentally that it is not just boosts that produce this kind of situation; passive spatial translations of the coordinate system can cause some sharp, discrete observables (such as angular momentum again, or coarse-grained position) to become unsharp.

There seems, in short, to be even more reason in quantum mechanics than in classical mechanics generally to regard sharp values of properties as relational, as opposed to intrinsic attributes of the system. This is not the end of the story, but in the meantime it is worth emphasising that the ways in which operators associated with distinct magnitudes transform under gauge or coordinate transformations can be quite different-ways which depend on the physical meaning of the magnitudes, and on their role within the quantum dynamics. The symmetry of the Hilbert space can look decidedly misleading when the implications of the symmetries of space and time are taken into account in quantum mechanics.

\section{Quantum frame bodies and relational realism.}

In the previous section we were considering magnitudes, such as position, momentum and angular momentum, defined relative to inertial coordinate systems. In practice, measurements of such magnitudes does not occur (except occasionally in an approximate sense). Actual measurements establish relations between bodies.

Consider a rigid, impenetrable box located somewhere in space; in nonrelativistic quantum mechanics it is a gauge- and coordinate-independent issue as to whether a quantum particle is wholly inside the box at any instant. The projection operator whose bivalent values correspond to the two possibilities ( 1 = 'wholly in', 0 = 'otherwise', say) must transform unitarily, so that if the 'in?' observable has a sharp value according to EEL relative to one gauge or frame, it has the same sharp value under the relevant transformation.

All this seems straightforward, but note that the role of the box is that of a classical body. What if we treat it quantum mechanically? Indeed, what are the implications for our present discussion if we treat the entire laboratory (even when construed as moving inertially) as a quantum mechanical system?

Let us imagine then an entire closed laboratory, denoted by $L$, replete with rulers and clocks rigidly fastened to its walls, along with other equipment. Inside the lab an investigation is taking place of the behaviour of some microsystem $p$; we denote the system comprising the laboratory minus the particle by $L_{-p}$. Let us further suppose that with respect to some 'external' inertial reference frame $F$, the state of the laboratory $L$ - which is assumed for simplicity to move freely-is at some instant of time an eigenstate of the $x$-component of total momentum relative to some Cartesian coordinate system adapted to $F$. (It might be imagined that this state is the result of an external measurement of the momentum of $L$ completed at that instant.) Indeed we 
might have it that $L$ is at rest relative to $F$. Given the additive nature of linear momentum, it can easily be shown ${ }^{41}$ that the (reduced) density matrix associated with the subsystem $p$ at the instant in question describes a mixture of eigenstates of the operator (defined in the $p$ factor Hilbert space) corresponding to the $x$-component of momentum. The particle is therefore wholly unlocalised relative to the $x$-axis of $F$. Hence it might seem that for an observer enclosed within the laboratory there is no possibility of 'seeing', or preparing, the particle as a localised wavepacket, concentrated in any specific region in the laboratory. 42

But note that at the instant in question $L$ is also wholly unlocalised, and it does not follow that relative to the rigid walls of $L_{-p}$ the particle $p$ cannot be considered strictly localised. Thus, the observer enclosed within $L$ can resort to a variable representing the momentum of $p$ relative to the much more massive system $L_{-p}$ (which acts as a momentum 'reservoir' in the apt terminology of Lubkin ${ }^{43}$ ); such a variable is, unlike the total momentum of $L$ relative to $F$, not strictly a conserved quantity. States associated with coherent superpositions of this 'relative momentum' can be effectively attributed to the particle $p$, which now represent localised wavepackets in the representation associated with the Fourier inverse of the relative momentum - the relative coordinate of the particle.

The upshot is that when the large system $L_{-p}$ is treated as a quantum frame body, meaningful localisation of the particle is possible even when $L$ is sharp in momentum relative to the external frame $F$. Observe that the 'laboratory frame' defined by $L_{-p}$ must be considered quite distinct from $F$, even when $L$ is at rest relative to $F$. Indeed, we might think of $F$ itself as being associated with, or modelled

\footnotetext{
${ }^{41}$ See Lubkin (1970).

42 This thought-experiment is of course highly idealised. No actual external measurement can determine the momentum of $L$ with absolute accuracy, and even if the intervention were to lead to significant, macroscopic indeterminacy in the position of the centre of mass of $L$, decoherence brought about by interaction of the system with the environment (if only the cosmological background radiation) would occur with extreme rapidity, resulting in a mixture of sufficiently localised states of the lab relative to $F$. But we are dealing here with a question of principle, and we may restrict out attention to the precise instant at which supposed de-localisation of $L$ occurs. Furthermore, if desired we could imagine an external measurement of the position of the laboratory, in which case it is the ability to create plane waves (momentum eigenstates) for the subsystem $p$ that would now be open to question.

43 See Lubkin (1970), where the analogous and more familiar problem of accounting for the localisability of the electron within a hydrogen atom is considered, when the atom as a whole is in a state of sharp momentum.
} 
by some separate, even larger quantum system (a momentum reservoir of astronomical proportions?) so that the 'conserved' total momentum of $L$ is now analogously considered a relative momentum defined with respect to this larger system.

The theory of 'quantum reference frames' has been developed further by Aharonov and Kauffher (1984), who posed a paradox (in one spatial dimension) based on our intuitions concerning the relativity principle. Ever since Galileo, we have come to expect that what transpires inside the inertial laboratory should not depend on its collective state of motion. In the context of quantum mechanics, an extension to this relativity (or 'equivalence') principle strongly suggests itself-one arguably implicit also in Lubkin's 1970 analysis: No observable processes occurring purely within $L$ should demonstrate whether or not the state of $L$ itself, relative to an external frame, is an eigenstate of centre-of-mass (c.o.m.) velocity or c.o.m. position. And yet the coordinate of the particle $p$ defined relative to $L_{-p}$ apparently fails to commute with the c.o.m. velocity of $L$ relative to the external frame $F$, as does the velocity of the particle $p$ relative to $L_{-p}$ with the c.o.m position of $L$, when the mass of $L_{-p}$ is finite.

The failure of these commutation relations rests, as Aharonov and Kauffher note, on the seemingly natural assumption that the relevant velocities of the free bodies $p, L_{-p}$, etc. are proportional to their respective canonical momenta. The authors further demonstrate that the difficulty is resolved if, for example, the particle $p$ feels the presence of a vector potential which, in their words, represents the 'kickback' of the finite mass reference frame. The particle's velocity relative to $L_{-p}$ is now proportional to its 'mechanical momentum' which of course depends on the mentioned vector potential as well as the canonical momentum. The vector potential is inversely proportional to the mass of $L_{-p}$ and depends also on the momentum of the external frame $F$ (itself also treated quantum mechanically) relative to $L_{-p}$. Although the vector potential in this case gives rise to no forces (as one would expect), when it is taken into account - as well as the analogous vector potential felt by $F$ - the desired commutation relations are restored, and the 'paradox of the quantum reference frame' is resolved.

The 1984 study of quantum reference frames by Aharanov and Kauffher goes considerably further than is indicated here, and certainly deserves more critical attention by philosophers of physics than it has received to date ${ }^{44}$. But the study is consistent with the present theme that direct measurements of observables like position, velocity and momentum involve establishing relations between the object

44 I am unaware of any analysis of this suggestive, but not wholly transparent study in the philosophical literature. 
system $p$ and some other material 'frame' body (such as $L_{-p}$ ), rather than an abstract coordinate system. This is not to say that inertial coordinate systems are not essential within the theoretical analysis; no accessible frame bodies with their attached physical clocks can strictly replace, or stand in for them, either in classical or quantum mechanics, except in an approximate sense ${ }^{45}$. Yet it is more realistic in relation to actual experiments to consider the observables under consideration as operationally defined in relation to such frame bodies. One lesson we may take from the Lubkin and Aharonov-Kauffher studies is that in standard quantum mechanics the particle $p$ may in principle have a sharp location, for instance, relative to $L_{-p}$, but no sharp location relative to an inertial coordinate system or (more to the point operationally) to an external frame body, at the same instant.

I think this point serves to provide more operational grounds for the view that emerged in the last section. If, in the hope of providing an ontological interpretation of quantum mechanics, we introduce state-dependent rules for assigning sharp values to magnitudes associated with a specific quantum system, we should recognise that the objective status of such sharp values is relational, not absolute. The full implications of this state of affairs for the standard formulation of the measurement problem in quantum mechanics (in which the sharp positions of the generic 'pointer' of the apparatus are normally required to be observer-independent elements of reality) are, perhaps, still not widely appreciated. ${ }^{46}$

\section{Acknowledgements.}

I wish to thank the referees for useful comments related to the original version of this paper, and Jeremy Butterfield for editorial improvements. I also thank Lucien Hardy, Peter Holland, Simon Saunders, Paul Teller and especially Guido Bacciagaluppi and Erik Sjöqvist for very helpful discussions, which prevented more misunderstandings than may be remaining.

45 Indeed, it is not clear to me that due recognition of the irreducible role of inertial coordinate systems in exactly defining the dynamics of quantum systems is given in Aharonov and Kauffher's 1984 analysis. (For further discussion of this role in the context of classical mechanics, see Barbour (1989, chapter 12) and Brown (1997, section 2).) It might for this reason be better to refer to the theory of quantum frame bodies, rather than quantum reference frames.

46 It may well be that the theory of quantum frame bodies is largely compatible with the interpretation of quantum mechanics defended recently in Mermin (1998), although I do not follow Mermin in his dismissal of both the measurement problem and the many-worlds interpretation on the basis of the wholly ineffable nature of consciousness from point of view of physics (op. cit. section VIII). 


\section{Appendix}

Consider a spacetime manifold equipped with a flat affine connection, and a curve $x^{i}(\lambda)$ associated with the affine parameter $\lambda$. Relative to an inertial coordinate system, the geodesic equation for this curve (i.e. the equation of motion of a free particle) takes the form of Newton's first law:

$$
\frac{\mathrm{d}^{2} x^{i}}{\mathrm{~d} \lambda^{2}}=0
$$

In analogy with the first part of the minimal coupling procedure outlined in Section $2 \mathrm{a}$, we wish to obtain the familiar generally covariant form of this equation,

$$
\frac{\mathrm{d}^{2} x^{i}}{\mathrm{~d} \lambda^{2}}+\Gamma_{k j}^{i} \frac{\mathrm{d} x^{k}}{\mathrm{~d} \lambda} \frac{\mathrm{d} x^{j}}{\mathrm{~d} \lambda}=0
$$

explicitly by way of replacing the ordinary partial derivative $\partial_{j}=\partial / \partial x^{j}$ by the covariant derivative associated with the connection coefficients $\Gamma_{k j}^{i}$, which may or may not be symmetric. Now let us write the vector field $\mathrm{d} x^{i} / \mathrm{d} \lambda$ defined on the curve in question as $V^{i}$; so (A1) becomes

$$
\frac{\mathrm{d} V^{i}}{\mathrm{~d} \lambda}=V^{j} \partial_{j} V^{i}=0
$$

Now replace the partial derivative $\partial_{j}$ in (A3) by the covariant derivative $\mathrm{D}_{j}$, which acts as $\mathrm{D}_{j} V^{i}=\partial_{j} V^{i}+\Gamma_{k j}^{i} V^{k}$. We then obtain

$$
V^{j}\left(\partial_{j} V^{i}+\Gamma_{k j}^{i} V^{k}\right)=0
$$

which is equivalent to (A2).

\section{Bibliography}

Aharonov, Y. and Anandan, J. (1987), 'Phase change during a cyclic quantum evolution',Physical Review Letters 58, 1593-1596.

Aharonov, Y. and Kauffher, T. (1984), 'Quantum frames of reference', Physical Review D 30, 368-385.

Aitchison, I.J.R. and Wanelik, K. (1992), Proceedings of the Royal Society of London A 439, 25-34. 
Anandan, J. (1980) 'On the hypotheses underlying physical geometry', Foundations of Physics 10, 601-629.

Anandan, J. (1989) 'Geometry of cyclic evolutions', in Bell's Theorem, Quantum Theory and Conceptions of the Universe, M. Kafatos (ed.), Kluwer Academic Publishers.

Anandan, J. (1991), 'A geometric approach to quantum mechanics', Foundations of Physics 21, 1265-1284.

Anandan, J. (1992), 'The geometric phase', Nature 360, 307-313.

Anandan, J. (1997), 'Reality and geometry in quantum theory', D. Phil. thesis in philosophy, Oxford University.

Anandan, J. and Brown, H.R. (1995). 'On the reality of space-time geometry and the wavefunction', Foundations of Physics 25, 349-360.

Bacciagaluppi, G. (1997), 'Making geometric phase both Galilean- and gaugeinvariant', manuscript.

Ballentine, L.E. (1990), Quantum Mechanics, Prentice Hall, New Jersey.

Barbour, J. (1989), Absolute or Relative Motion? Vol I: The Discovery of Dynamics, Cambridge University Press.

Berry, M.V. (1984), 'Quantal phase factors accompanying adiabatic changes', Proceedings of the Royal Society of London A 392, 45-57.

Berry, M.V. and Robbins, J.M. (1997), 'Indistinguishability for quantum particles: spin, statistics and the geometric phase', Proceedings of the Royal Society of London A 453, 1771-1790.

Brown, H.R. (1996), 'Bovine metaphysics: remarks on the significance of the gravitational phase effect in quantum mechanics', in Persepectives on Quantum Reality, R. Clifton (ed.), Kluwer Academic Publishers; pp. 183-193.

Brown, H.R. (1997), 'On the role of special relativity in general relativity', International Studies in the Philosophy of Science 11, 67-81.

Brown, H.R., Elby, A. and Weingard, R. (1996), 'Cause and effect in the pilot-wave intrepretation of quantum mechanics', in Bohmian Mechanics and Quantum Theory: An Appraisal, J.T. Cushing et al. (eds.), Kluwer Academic Publishers; pp. 309-319.

Brown, H.R. and Holland, P.R. (1998), 'The Galilean covariance of quantum mechanics in the case of external fields'; to appear in American Journal of Physics.

Brown H.R., Sjöqvist, E. and Bacciagaluppi, G. (1998), 'Remarks on identical particles in de Broglie-Bohm theory', to appear in Physics Letters A..

Brown H.R., Suárez, M. and Bacciagaluppi, G. (1998), 'Are "sharp values" of observables always objective elements of reality?', in The Modal Interpretation 
of Quantum Mechanics, D Dieks and P Vermaas (eds.), Kluwer Academic Publishers; pp. 289-306.

Brown, H.R. and Sypel R. (1995). 'On the meaning of the relativity principle and other symmetries', International Studies in the Philosophy of Science 43, 381407.

Collins, P.D.B., Martin, A.D. and Squires, E.J. (1989), Particle Physics and Cosmology, John Wiley \& Sons, New York.

Fonda, L. and Ghirardi, G.L. (1970), Symmetry Principles in Quantum Physics, Marcel Dekker, New York.

García de Polavieja, G. (1997), 'Galilean invariant structure of geometric phase', Physics Letters A 232, 1-3.

García de Polavieja, G. and Sjöqvist, E. (1998), 'Externding the quantal adiabatic theorem: Geometry of noncyclic motion', American Journal of Physics 66, 431438.

Holland, P.R. (1993), The Quantum Theory of Motion, Cambridge University Press.

Jauch, J.M. (1964), 'Gauge Invariance as a Consequence of Galilei-Invariance for Elementary Particles', Helvetica Physica Acta 37, 284-292.

Kuchar , K. (1980), 'Gravitation, geometry, and nonrelativistic quantum theory', Physical Review D 22, 1285-1299.

Lawrie, I.D. (1990), A Unified Grand Tour of Theoretical Physics, Adam Hilger (IOP Publishing Ltd.).

Le Bellac, M. and Lévy-Leblond, J.-M. (1973), 'Galilean Electromagnetism', Il Nuovo Cimento 14B, 217-233.

Leinaas, J.M. and Myrheim, J. (1977), 'On the theory of identical particles.', Nuovo Cimento B 37, 1-23.

Lévy-Leblond J.-M. (1976), 'Quantum Fact and Fiction: Clarifying Landé's Pseudoparadox', American Journal of Physics 44, 1130-2.

Lubkin, E. (1970), 'On violation of superselection rules', Annals of Physics 56, 69-80.

Mermin, N.D. (1998). 'What is quantum mechanics trying to tell us?', American Journal of Physics 66, 753-767.

Mukunda, N. and Simon, R. (1993), "Quantum kinematic approach to the geometric phase. I. General formalism', Annals of Physics 228, 205-268.

Nakahara, M. (1990), Geometry, Topology and Physics, Adam Hilger, IOP Publishing Ltd, Bristol.

Rai Dastidar, T.K. and Rai Dastidar, K. (1994), 'Gauge Invariance in Non-Relativistic Quantum Mechanics', Il Nuovo Cimento 109B, 1115-1118.

Rai Dastidar, T.K. and Rai Dastidar, K. (1995), 'Local gauge invariance of relativistic quantum mechanics and classical relativistic fields', Modern Physics Letters A 10, 1843-1846. 
Ryder, L.H. (1987), Quantum Field Theory, Cambridge University Press (paperback edition).

Sakurai, J.J. (1964), Invariance Principles and Elementary Particles, Princeton University Press.

Sjöqvist, E., Brown, H.R. and Carlsen, H. (1997) 'Galilean noninvariance of geometric phase', Physics Letters A 229, 273-278.

Takagi, S. (1990), 'Equivalence of a harmonic oscillator to a free particle', Progress of Theoretical Physics, 84, 1019-1024.

Takagi, S. (1991), 'Quantum dynamics and non-inertial frames of reference, I, II and III', Progress of Theoretical Physics 85, 463-479, 723-742; 86, 783-798.

Teller, P. (1997), 'Essay Review. A Metaphysics for Contemporary Field Theories', Studies in History and Philosophy of Modern Physics 28, 507-522..

Valentini, A. (1997), 'On Galilean and Lorentz invariance in pilot-wave dynamics', Physics Letters A 228, 215-222.

Wald, R.M. (1984), General Relativity, University of Chicago Press. 